\title{
A psychometric analysis and revalidation of the Yale-Brown Obsessive Compulsive Scale modified for Binge Eating in adults with binge eating disorder
}

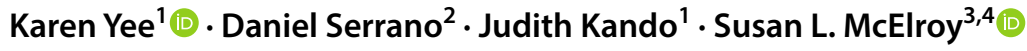

Accepted: 16 August 2019 / Published online: 31 August 2019

(c) The Author(s) 2019

\begin{abstract}
Purpose The Yale-Brown Obsessive Compulsive Scale modified for Binge Eating (Y-BOCS-BE) assesses the obsessiveness of binge eating thoughts and compulsiveness of binge eating behaviors. The findings of this study extend previously published Y-BOCS-BE psychometric evaluations in adults with binge eating disorder (BED).

Methods Data from three phase 3 lisdexamfetamine dimesylate studies in adults with BED (two randomized, double-blind, placebo-controlled short-term efficacy studies; one double-blind, placebo-controlled, randomized-withdrawal maintenanceof-efficacy study) were used. Psychometric evaluations included assessment of Y-BOCS-BE dimensionality, internal consistency, convergent validity, test-retest reliability, and determinations of clinically meaningful improvement using distribution- and anchor-based methods.

Results Domain specification analyses determined that the Y-BOCS-BE possessed a bifactor structure composed of a general binge eating severity domain and three subdomains (obsessive/compulsive, restraint, and control). Y-BOCS-BE internal consistency was maximized at week 12 (Cronbach's $\alpha, 0.95$ ) and test-retest reliability was maximized in the 8-week retest interval from week 4 to week 12 across all no-change anchors $(r=0.74-0.90)$. Likewise, convergent validity of the Y-BOCS$\mathrm{BE}$ across all validators was maximized at week 12 (all $r \geq 0.66$ ). Meaningful improvement for Y-BOCS-BE total scores was estimated to require score reductions of 12 to 17 points depending on the anchor.

Conclusions The Y-BOCS-BE is a valuable tool for assessing BED symptoms. Maximization of Y-BOCS-BE reliability and validity at later study time points may be related to both treatment effects and improved insight into BED by participants during the study.
\end{abstract}

Keywords Binge eating disorder · Calibration · Psychometrics · Reliability · Yale-Brown Obsessive Compulsive Scale modified for Binge Eating

Judith Kando-Formerly of Shire, a member of the Takeda group of companies, Lexington, MA, USA.

Electronic supplementary material The online version of this article (https://doi.org/10.1007/s11136-019-02277-8) contains supplementary material, which is available to authorized users.

\section{Karen Yee}

karen.yee@takeda.com

1 Shire, a member of the Takeda group of companies, Lexington, MA, USA

2 Pharmerit International, Bethesda, MD, USA

3 Lindner Center of HOPE, Mason, OH, USA

4 University of Cincinnati College of Medicine, Cincinnati, OH, USA

\section{Introduction}

The Yale-Brown Obsessive Compulsive Scale modified for Binge Eating (Y-BOCS-BE), which assesses the obsessiveness of binge eating (BE) thoughts and compulsiveness of BE behaviors [1], is a modified version of the Yale-Brown Obsessive Compulsive Scale [2]. The Y-BOCS-BE has been used in studies of binge eating disorder (BED) to assess the efficacy of pharmacotherapy [3-6]. Across studies, reductions in BE were accompanied by statistically significant reductions in Y-BOCS-BE total score [3-6].

Psychometric testing and analysis of the Y-BOCS-BE is being conducted as a multi-stage process to optimize the characterization of BED. A preliminary validation of the Y-BOCS-BE [1] was conducted using data from a phase 2 
study of lisdexamfetamine dimesylate (LDX) in adults with moderate-to-severe BED [7]. This analysis demonstrated that the Y-BOCS-BE had high internal consistency (Cronbach's $\alpha, 0.81$ ) at baseline [1]. The Y-BOCS-BE also exhibited good construct validity in relation to multiple reference measures, including the Three-Factor Eating Questionnaire (TFEQ) and Binge Eating Scale (BES), but correlations with reference measures at baseline were lower than for score changes from baseline to end-of-study [1]. The estimated range of Y-BOCS-BE total score reductions indicative of minimal clinically important change was 4 to 17 points [1]. These data suggested the Y-BOCS-BE is a reliable and valid measure of treatment benefit in BED [1].

This study extends the preliminary psychometric validation of the Y-BOCS-BE by using two phase 3 trials in adults with BED for validation and another phase 3 trial for characterization of treatment benefit on developed scores [6, 8]. LDX was superior to placebo in reducing BE days/week in the two short-term efficacy studies [6] and in prolonging time to relapse in the randomized-withdrawal maintenanceof-efficacy study [8]. The Y-BOCS-BE was included as a secondary efficacy endpoint in each of the studies. In the short-term efficacy studies, LDX was superior to placebo in reducing Y-BOCS-BE total score [6]. In the maintenanceof-efficacy study, LDX maintained Y-BOCS-BE total score reductions during the randomized-withdrawal phase [8]. These analyses further examine the dimensionality, itemlevel properties, scoring, and minimal clinically important improvement (MCII) of the Y-BOCS-BE and LDX treatment effects on Y-BOCS-BE scores from the aforementioned studies.

\section{Methods}

\section{Study design and participants}

Three multicenter LDX clinical studies, the designs of which have been described $[6,8]$, were used in these analyses. The short-term efficacy studies were identically designed, 12-week, randomized, double-blind, placebo-controlled studies (ClinicalTrials.gov, NCT01718483 [study 1] and NCT01718509 [study 2]) [6]. The maintenance-of-efficacy study was a double-blind, placebo-controlled, randomizedwithdrawal study (ClinicalTrials.gov, NCT02009163 [study $3]$ ), with a 12-week open-label phase and 26-week doubleblind, randomized-withdrawal phase [8].

Comprehensive inclusion and exclusion criteria have been published [6, 8]. In summary, all studies enrolled men and nonpregnant women (age, 18-55 years; body mass index [BMI], $\geq 18$ to $\leq 45 \mathrm{~kg} / \mathrm{m}^{2}$ ). All participants met Diagnostic and Statistical Manual of Mental Disorders, Fourth Edition, Text Revision, BED criteria and had protocol-defined moderate-to-severe BED ( $\geq 3 \mathrm{BE}$ days/week for 14 days before baseline and Clinical Global Impressions-Severity [CGI-S] ratings $\geq 4$ at screening and baseline). Key exclusion criteria included current anorexia nervosa or bulimia nervosa diagnoses; current comorbid Axis I or Axis II psychiatric disorders controlled with prohibited medications or uncontrolled and associated with significant symptoms; a history of cardiovascular health problems; clinically significant electrocardiogram abnormalities at screening; and moderate or severe hypertension.

For all studies, protocols were approved by ethics committees (see Supplemental Table 1 for a complete listing) and conducted in accordance with International Conference on Harmonisation Good Clinical Practice and the principles of the Declaration of Helsinki. All study participants were required to provide written, informed consent before entering the studies.

\section{Measures}

The Y-BOCS-BE [1] is a 10-item clinician-rated scale $(0=$ no symptoms to $4=$ extreme symptoms $)$; total score ranges from 0 to 40 . The items assess the obsessiveness of $\mathrm{BE}$ thoughts (time occupied by thoughts about BE; interference due to thoughts about $\mathrm{BE}$; distress associated with thoughts about $\mathrm{BE}$; resistance of thoughts about BE; degree of control over thoughts about $\mathrm{BE}$ ) and $\mathrm{BE}$ behaviors (time spent on compulsive behaviors about BE; interference due to $\mathrm{BE}$; distress associated with $\mathrm{BE}$; resistance to $\mathrm{BE}$; degree of control over BE). In these studies, the Y-BOCS-BE was assessed at baseline and weeks 4,8 , and 12/early termination (ET) in the short-term efficacy studies [6] and at open-label baseline and weeks 4, 12 (randomized-withdrawal baseline [RWB]), 16, 20, 24, 28, 32, 36, and 38/ET in the maintenance-of-efficacy study [8].

Several validated instruments were used as reference measures. The 16-item self-report BES [9] assesses behavioral, affective, and attitudinal components of the subjective BE experience; impulsivity and compulsivity are independently assessed. The BES was assessed at baseline and weeks 4,8 , and 12/ET in the short-term efficacy studies. The 51-item self-reported TFEQ [10] assesses three dimensions of eating behavior: cognitive restraint, perceived hunger, and emotionally based disinhibition of eating. The TFEQ was assessed at baseline and weeks 4, 8, and 12 in the short-term efficacy studies. The CGI-S and Clinical Global Impressions-Improvement (CGI-I) scales [11] evaluate the severity of BED symptoms and symptom improvement over time. The CGI-S was rated from 1 (normal, not at all ill) to 7 (among the most extremely ill); the CGI-I was rated from 1 (very much improved) to 7 (very much worse). In all studies, the CGI-S was assessed at all study visits and the CGI-I was assessed at all post-baseline visits. Study participants 
also recorded $\mathrm{BE}$ in a daily diary that was collected at each study visit.

\section{Psychometric evaluations}

Psychometric modeling was conducted using Mplus ${ }^{\circledR}$ version 8 for exploratory factor analysis (EFA) estimation and flexMIRT ${ }^{\circledR}$ version 3 (Vector Psychometric Group, Chapel Hill, NC) for estimation of item response theory (IRT) models. Estimates of reliability, validity, meaningful improvement, and treatment efficacy were conducted with $\mathrm{SAS}^{\circledR}$ version 9.4 (SAS Institute Inc., Cary, NC). There were no missing data at baseline where psychometric models were estimated. Therefore, no missing data handling methods were required or applied to any of the latent variable measurement models used in this study. The maximum proportion of missing data post baseline occurred at week 4, but was less than $1 \%$ ( 3 of 313 subjects, or $0.96 \%$ ). Therefore, consistent with the trial justification that such negligible missing data rates are largely inconsequential [12], no missing data techniques were used beyond complete case.

\section{Descriptive Y-BOCS-BE evaluations}

Item response distributions were evaluated by examining the response frequencies for each item at baseline: the sparseness of response option endorsement and floor and ceiling effects (operationally defined as a threshold of $20 \%$ of participants endorsing the lowest or highest response option). Inter-item polychoric correlations were estimated at baseline across all items to evaluate provisional domain patterns and identify item redundancies.

\section{Y-BOCS-BE domain structure}

Although the original validation study examined the domain structure of the Y-BOCS-BE [1], the domain structure was re-examined in this manuscript using the two short-term efficacy studies of LDX described above. Domain specification followed a two-stage procedure in which evidence obtained from both EFA and IRT was used to arrive at an empirically justifiable domain solution. Selection of the final domain solution was based upon balancing model fit, clinical input, interpretability of the solution, and scoring statistics [13]. To control for the risk that the two-stage procedure might arrive at a solution due either to over-fitting or capitalization on sample-specific characteristics [14], the solution obtained from study 1 was independently replicated in study 2 . The reproducibility of the solution was compared between the two studies to determine whether the solution was stable [15]. Model fit indices for EFA model selection were obtained from limited information estimation. Specifically, the mean and variance adjusted weighted least squares
(WLSMV) estimator was used. Reported EFA item parameter estimates, item response theory (IRT) model fit, and IRT item parameters were all obtained from full-information marginal maximum likelihood estimation. Details of the modern psychometric methods are given in the Supplemental Materials. Two competing domain models were examined. One model was a MIRT with domains equal to those obtained from the final EFA solution, and the other was a bifactor model [16], which used the same domain structure as the MIRT augmented by a general domain reflecting overall BED severity.

After agreement on the interpretability and clinical relevance of the best fitting solution was achieved, scoring statistics were computed at baseline to determine how best to use the Y-BOCS-BE to characterize binge eating severity. Scoring statistics computed at baseline compared whether domain scoring (based on the MIRT) or a total score (based on the bifactor) was more appropriate for characterizing binge eating severity. Scoring statistics employed included the $\omega, \omega_{\mathrm{H}}$, the ratio of $\omega_{\mathrm{H}}$ to $\omega$, the explained common variance (ECV), and the $H$ statistic [13].

\section{Y-BOCS-BE scoring, reliability, validity}

Reliability, validity, and meaningful change were estimated for Y-BOCS-BE sum scores in studies 1 and 2. Internal consistency was assessed at baseline using Cronbach's $\alpha$, with estimates $\geq 0.7$ considered acceptable. Test-retest reliability was estimated via intra-class correlation coefficients with two retest intervals: baseline to week 4 and week 4 to week 12 conditioned upon no change on anchor variables (CGI-I and CGI-S). Concurrent validity was estimated via Spearman correlations at baseline, week 4 , and week 12 between Y-BOCS-BE scores and binge days, TFEQ domains (cognitive, disinhibition, and hunger), and BES total score.

\section{Y-BOCS-BE clinically meaningful improvement and efficacy}

Meaningful change was computed on Y-BOCS-BE change scores computed between baseline and week 12 using both distribution- and anchor-based estimates of minimal clinically important improvement (MCII). Distribution-based estimates included 1 and 0.5 baseline standard deviation (SD) and baseline standard error of the measurement (SEM). In addition, following Harvill 1991 [17], the baseline SD and SEM were used to index the amount an individual needed to improve beyond the average change score $(\Delta \mu)$ to reflect meaningful change. These difference estimates are noted as $\Delta \mu-$ Baseline SD and $\Delta \mu-$ Baseline SEM, respectively. Anchor-based MCII was estimated by comparing Y-BOCS$\mathrm{BE}$ change score point and interval estimates for minimal improvement defined by the CGI-S (1-point improvement between baseline and week 12) and CGI-I (response 
category 3, with descriptor of "minimally improved"). In addition to point estimates, cumulative distribution functions (CDFs) stratifying on CGI-I and CGI-S were plotted; separation of CDFs at the meaningful change estimate location between treatment arms was tested.

Mixed-effects models for repeated measures (MMRM) were used to assess treatment efficacy using Y-BOCS-BE change from baseline scores based on the full analysis set (FAS), as defined for each study [6, 8]. An unstructured error covariance matrix was used for the repeated measures. Efficacy estimates were based on least squares (LS) mean contrasts at each post-baseline visit. The MMRM was adjusted for baseline Y-BOCS-BE scores and the baseline Y-BOCS$\mathrm{BE}$ score by treatment interaction.

For the maintenance-of-efficacy study (study 3), efficacy analyses differed slightly owing to the randomized-withdrawal study design. Y-BOCS-BE scores obtained during the randomized-withdrawal period were modeled as outcomes in a linear mixed model, with the primary predictors of time, treatment, and treatment by time. Covariate adjustments included the RWB Y-BOCS-BE scores and time by RWB score interaction. An unstructured residual covariance matrix was used in this model.

\section{Results}

\section{Participant demographics and disposition}

Participant disposition and demographics have been described in detail elsewhere [6, 8]. In brief, the FAS included the following number of participants: study 1 , $n=374$ (LDX, $n=190$; placebo, $n=184$ ); study $2, n=350$ (LDX, $n=174$; placebo, $n=176$ ); and study $3, n=267$ (LDX, $n=136$; placebo, $n=131$ ). Across studies, most participants were white (74.0-84.3\%) and were women (86.3-87.6\%); mean \pm SD age and BMI, respectively, ranged from $38.0 \pm 10.04$ to $38.7 \pm 10.03$ years and $33.43 \pm 6.245$ to $33.89 \pm 6.050 \mathrm{~kg} / \mathrm{m}^{2}$. Mean $\pm \mathrm{SD}$ duration of exposure (in days) was $75.7 \pm 20.81$ with LDX and 76.6 \pm 20.72 with placebo for study $1 ; 75.8 \pm 20.14$ with LDX and 73.1 \pm 22.99 with placebo for study 2 ; and $157.6 \pm 51.60$ with LDX and $98.9 \pm 72.83$ with placebo during the randomized-withdrawal phase of study 3 .

\section{Psychometric evaluations of the Y-BOCS-BE}

\section{Descriptive evaluations}

The missing data rate was negligible (baseline, $0 \%$; week $4,0.96 \%$; week $12,0.32 \%$ ). Item distribution analyses indicated there were no floor or ceiling effects. Inter-item correlations were weak at baseline (range, 0.0-0.70), with only two correlations exceeding 0.6 , and were acceptable at week 12 (range, 0.62-0.88).

\section{Dimensionality assessments}

Model fit indices indicated that the Y-BOCS-BE domain structure was optimally characterized with a three-factor model interpreted after an oblique quartimax rotation $\left(\chi^{2}\right.$ $P$ value $<0.0001 ;$ RMSEA $[95 \% \mathrm{CI}]=0.09$ [0.07-0.11]; $\mathrm{CFI}=0.98 ; \mathrm{TLI}=0.94$; $\mathrm{SRMR}=0.04)$; this structure was carried forward into the IRT model estimation. The three domains of obsessive/compulsive, restraint, and control were defined, respectively, by time spent on, disruption of life due to, and distress associated with BE thoughts and actions (items 1-3, 6-8); ability to resist BE thoughts and actions (items 4 and 9); and ability to control BE thoughts and actions (items 5 and 10). These domains were modestly correlated ( $r=0.24,0.22,0.32$, respectively).

Because this three-domain structure differed from both the two- and seven-domain solutions reported by Deal et al. [1], confirmatory analyses were performed to assess whether the three-domain structure fits better than the parsimonious two-domain structure reported by Deal et al. [1]. These structures were compared to a bifactor modification to the three-domain structure, which was found to achieve the best fit across all indices (C2 $P$ value $=0.0001$; RMSEA [95\% $\mathrm{CI}]=0.09$ [0.07-0.11]; $\mathrm{AIC}=8394.36 ; \mathrm{BIC}=8630.14)$ and minimize local dependence. The bifactor solution, emphasizing measurement of BE severity in a total domain, was clinically determined to also possess the greatest verisimilitude and interpretability. Consequently, the bifactor structure was retained in the IRT model estimation. A full explication of the evidence supporting this conclusion is contained in the Supplemental Materials.

Figure 1 depicts the test information function (TIF; proportional to scale reliability) for the final IRT model solution. The TIF is multimodal, with score reliability maximized at approximately $1 \mathrm{SD}$ above and below the mean, which is identified by 0 on the $\mathrm{x}$-axis, for the latent $\mathrm{BE}$ severity scale.

\section{Scoring, reliability, and validity}

Baseline scoring statistics supported the use of total score $\left(\omega=0.94 ; \omega_{\mathrm{H}}=0.89 ; \omega_{\mathrm{H}} / \omega=0.95 ; \mathrm{ECV}=0.95 ;\right.$ and $\mathrm{H}=0.87$ ). Definitions and interpretations of these statistics are given in Rodriguez et al. [13]. Descriptive statistics characterizing the distributions of these scores for baseline, week 4, and week 12 are presented in Table 1, and reflect the substantial reductions in post-baseline scores.

Table 2 summarizes internal consistency reliability at baseline and week 12. Y-BOCS-BE item to total correlations and item $\alpha$ were lower at baseline for each item (ranges: 
Fig. 1 Final IRT model TIF solution. IRT item response theory, TIF test information function, $\varnothing$ latent binge eating severity

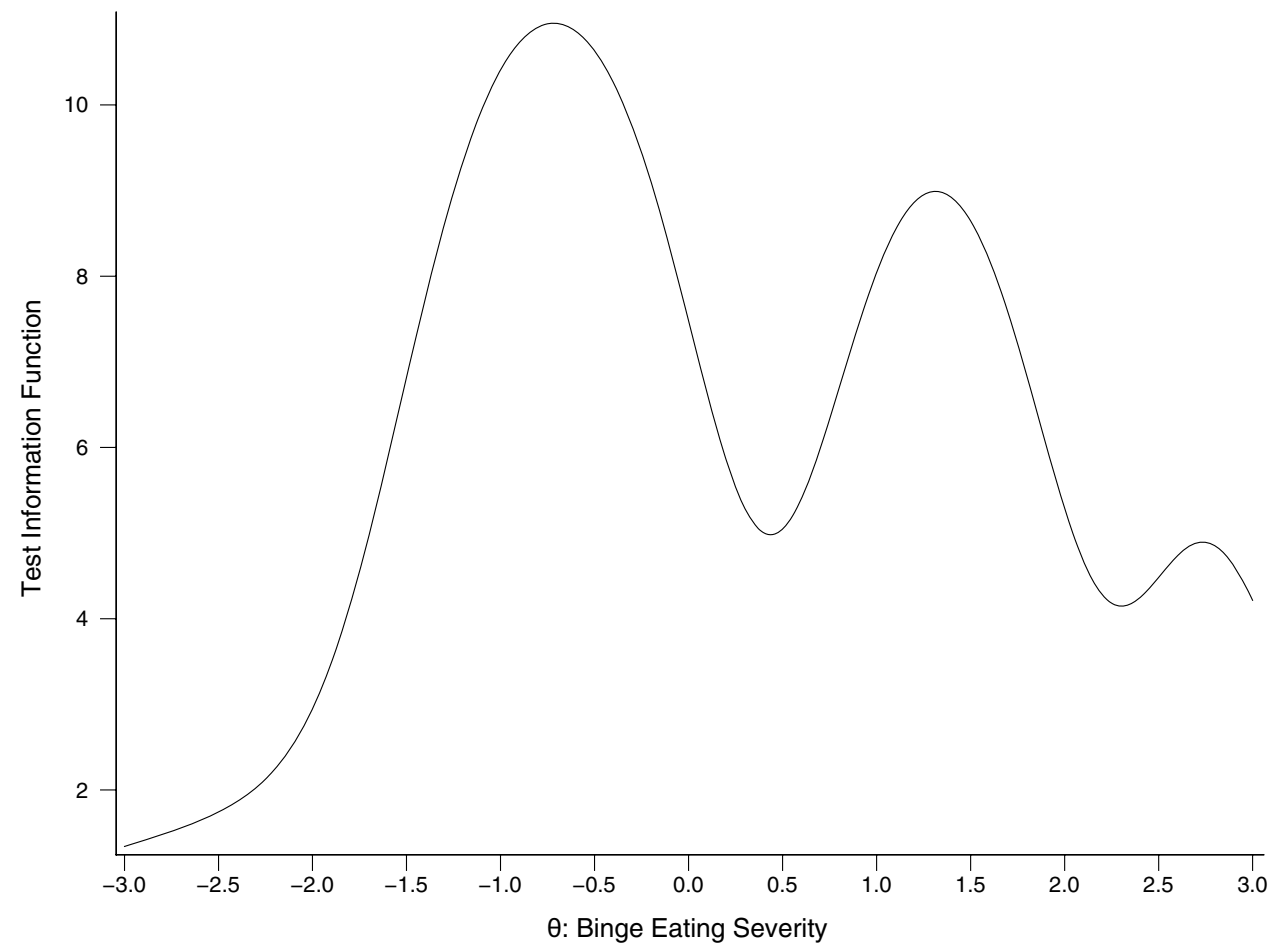

Table 1 Y-BOCS-BE score descriptive statistics short-term efficacy studies stratified by visit

\begin{tabular}{llrrll}
\hline Visit & $n$ & Mean & SD & $\begin{array}{l}\text { Minimum } \\
\text { value }\end{array}$ & $\begin{array}{l}\text { Maxi- } \\
\text { mum } \\
\text { value }\end{array}$ \\
\hline Baseline & 313 & 21.7 & 4.9 & 9 & 39 \\
Week 4 & 310 & 10.3 & 8.3 & 0 & 31 \\
Week 12 & 313 & 9.5 & 8.2 & 0 & 33 \\
\hline
\end{tabular}

$S D$ standard deviation, $Y$-BOCS-BE Yale-Brown Obsessive Compulsive Scale modified for Binge Eating correlations, 0.20-0.56; Cronbach's $\alpha, 0.74-0.78$ ) and for total score (Cronbach's $\alpha, 0.77$ ) than at week 12 for each item (ranges: correlations, 0.74-0.85; Cronbach's $\alpha$, all 0.95) and total score (Cronbach's $\alpha, 0.96)$. However, baseline total score internal consistency achieved the pre-specified acceptability criterion.

Test-retest reliability correlations between baseline and week 4 in participants whose CGI-S ratings did not change between assessments or who had CGI-I ratings of "no change" between assessments were low $(r=0.34$ and

Table 2 Y-BOCS-BE internal consistency estimates at baseline and week 12

\begin{tabular}{|c|c|c|c|c|c|c|}
\hline & \multicolumn{2}{|c|}{ Item:total correlation } & \multicolumn{2}{|c|}{ Item-level Cronbach's $\alpha$} & \multicolumn{2}{|c|}{$\begin{array}{l}\text { Y-BOCS-BE total score } \\
\text { Cronbach's } \alpha\end{array}$} \\
\hline & Baseline & Week 12 & Baseline & Week 12 & Baseline & Week 12 \\
\hline Y-BOCS-BE Item 1: binge thought time & 0.48 & 0.81 & 0.75 & 0.95 & NA & NA \\
\hline Y-BOCS-BE Item 2: binge thought disruption & 0.56 & 0.76 & 0.74 & 0.95 & NA & NA \\
\hline Y-BOCS-BE Item 3: binge thought distress & 0.52 & 0.81 & 0.74 & 0.95 & NA & NA \\
\hline Y-BOCS-BE Item 4: resist thoughts & 0.42 & 0.76 & 0.76 & 0.95 & NA & NA \\
\hline Y-BOCS-BE Item 5: control thoughts & 0.46 & 0.84 & 0.75 & 0.95 & NA & NA \\
\hline Y-BOCS-BE Item 6: binge time & 0.38 & 0.82 & 0.76 & 0.95 & NA & NA \\
\hline Y-BOCS-BE Item 7: binging disrupt & 0.53 & 0.80 & 0.74 & 0.95 & NA & NA \\
\hline Y-BOCS-BE Item 8: binging distress & 0.47 & 0.85 & 0.75 & 0.95 & NA & NA \\
\hline Y-BOCS-BE Item 9: resist binge & 0.20 & 0.74 & 0.78 & 0.95 & NA & NA \\
\hline Y-BOCS-BE Item 10: control binge & 0.35 & 0.85 & 0.77 & 0.95 & NA & NA \\
\hline Y-BOCS-BE total score & NA & NA & NA & NA & 0.77 & 0.96 \\
\hline
\end{tabular}

$N A$ not applicable, $Y$-BOCS-BE Yale-Brown Obsessive Compulsive Scale modified for Binge Eating 
$r=0.59)$. Sensitivity analyses of test-retest reliability correlations between week 4 and week 12, conditioned on similar CGI-S and CGI-I anchors, were acceptably higher $(r=0.77$ and $r=0.90$ ).

At baseline, convergent validity estimates were weak $(r \leq 0.4)$ across all validators (Table 2$)$. At week 4 , convergent validity estimates increased for the TFEQ disinhibition and hunger domains but remained low for binge days and the TFEQ cognitive domain (Table 3). Convergent validity estimates were high for all validators (Table 3 ; all $r \geq 0.66$ ) at week 12 except for binge days and the TFEQ cognitive domain.

The discussion section contains an extensive clinical explanation of the relationship between poor BED insight and self-monitoring effects, addressing the low test-retest reliability and convergent validity.

\section{Clinically meaningful improvement and efficacy}

Distribution-based MCII estimates for $\Delta \mu$-Baseline SD and $\Delta \mu$-Baseline SEM, respectively, were -17.04 and -14.48 in study 1 and -15.06 and -12.67 in study 2 . Anchor-based score estimates for "minimal improvement" on the CGI-I or a 1-point improvement on the CGI-S from baseline to week 12 , respectively, were -5.00 and -6.78 in study 1 and -4.52 and -5.63 in study 2 . The proportions of participants achieving the anchor-based estimates were excessively high $(>80 \%)$. Therefore, the distribution-based estimates $(\Delta \mu$-Baseline SD and $\Delta \mu$-Baseline SEM) reported above were retained.

Observed treatment-stratified CDF curves with estimated odds ratios corresponding to estimated MCII values are shown in Fig. 2. MCII estimates based on $\Delta \mu$-Baseline SD or $\Delta \mu$-Baseline SEM met criteria for optimal MCII for scores (Fig. 2A). Fifty percent of participants in the LDX group and $16.6 \%$ in the placebo group achieved a degree of improvement that met or exceeded the $\Delta \mu$-Baseline SD estimate; $63.5 \%$ of participants in the LDX group and $28.0 \%$ in the placebo group achieved a degree of improvement that

Table 3 Convergent validity estimates (Spearman correlations) with Y-BOCS-BE scores at baseline, week 4, and week 12

\begin{tabular}{lccc}
\hline Validator & Baseline & Week 4 & Week 12 \\
\hline Binge days & 0.24 & 0.08 & 0.01 \\
TFEQ cognitive domain & -0.05 & -0.15 & -0.14 \\
TFEQ disinhibition domain & 0.11 & 0.60 & 0.72 \\
TFEQ hunger domain & 0.09 & 0.62 & 0.72 \\
BES total score & 0.27 & ND & 0.77 \\
\hline
\end{tabular}

$B E S$ Binge Eating Scale, $N D$ not determined, TFEQ Three-Factor Eating Questionnaire, $Y$-BOCS-BE Yale-Brown Obsessive Compulsive Scale modified for Binge Eating

${ }^{\mathrm{a}}$ Analyses not conducted on week 4 data for the BES met or exceeded the $\Delta \mu$-Baseline SEM estimate. Corresponding CDF curves for study 2 (Fig. 2B) also showed substantial separation between treatment groups.

LDX treatment effects across all studies are summarized in Table 4. The LS mean treatment difference for change from baseline (LDX vs placebo) significantly favored LDX over placebo at weeks 4,8 , and 12 (all $P<0.0001$ ) in studies 1 and 2; these findings are consistent with the primary publication [6]. The LS mean treatment difference for change from randomized-withdrawal baseline (LDX vs placebo) significantly favored LDX over placebo at weeks 16, 20, 24, 28,32 , and 38 (all $P<0.0001$ ) in study 3 , which is consistent with the primary publication [8].

\section{Discussion}

This study described the psychometric evaluation of the Y-BOCS-BE based on phase 3 clinical data from two independently conducted and identically designed short-term efficacy studies and a maintenance-of-efficacy study of LDX in adults with BED. These analyses demonstrated that the Y-BOCS-BE possesses a bifactor structure composed of a general binge eating severity domain and three subdomains (obsessive/compulsive, restraint, and control) and exhibits strong internal consistency. Test-retest reliability from baseline to week 4 and convergent validity were poor at baseline but substantially better from week 4 to week 12 for test-retest reliability and at week 12 for convergent validity, potentially due to LDX treatment effects and improved insight into BED at later points during the studies. MCII estimators based on raw metrics displayed considerable heterogeneity, which was reduced with score standardization. Estimated treatment effects were significant, consistent with published results $[6,8]$, and accounted for a substantial percentage of the variance in change scores.

The Y-BOCS-BE measured BED severity via items assessing $\mathrm{BE}$ thoughts and actions. These analyses demonstrated that the Y-BOCS-BE can be decomposed into three domains (obsessive/compulsive [6 items], restraint [2 items], and control [2 items]) rather than the hypothesized two domains (obsessiveness of binge thoughts, compulsiveness of binge actions) [1]. This is consistent with previously published findings, which also reported that a two-domain structure did not adequately describe the Y-BOCS-BE [1]. In these analyses, a bifactor solution of the three-domain structure fits the data optimally and was carried forward. Under the bifactor solution, subdomains are assumed to arise because of idiosyncratic effects, such as shared item phrasing, that are assumed to be noise and best explained by the common unidimensional domain of BED severity.

Inter-item correlations, internal consistency, test-retest reliability, and convergent validity were poor for analyses 
Fig. 2 CDF curves for the Y-BOCS-BE with modeled ORs imposed at MCII locations. Panels $\mathbf{A}$ and $\mathbf{B}$ : Based on sum scores in studies 1 and 2, respectively. $B L$ baseline, $C D F$ cumulative distribution function, $M C I I$ minimal clinically important improvement, $O R$ odds ratio, $S D$ standard deviation, $S E M$ standard error of measurement, $L D X$ lisdexamfetamine dimesylate, $Y$-BOCS$B E$ Yale-Brown Obsessive Compulsive Scale modified for Binge Eating

\section{A Study 1 - Sum Score CDFs}

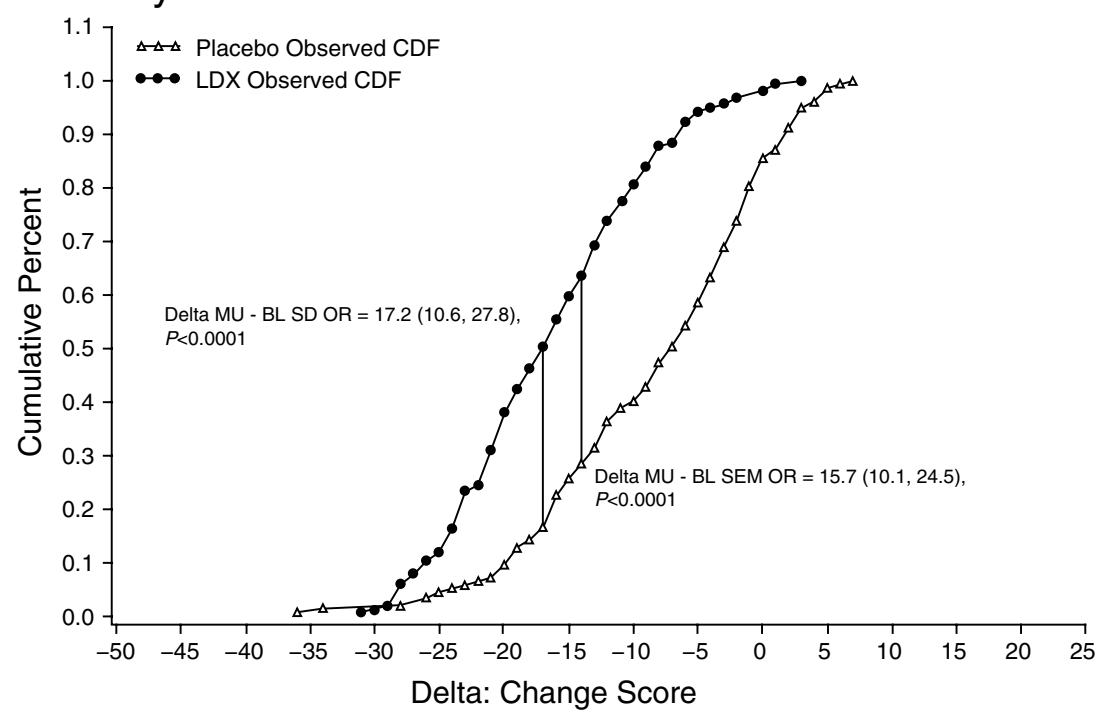

\section{B Study 2 - Sum Score CDFs}

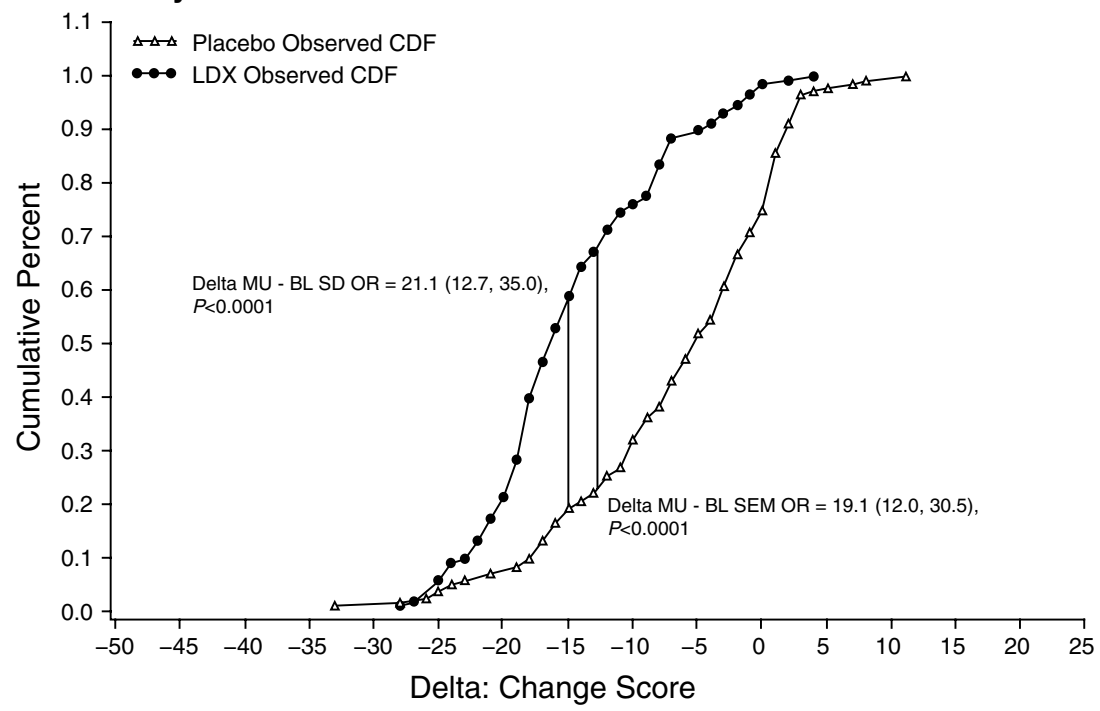

conducted at baseline but substantially improved at later time points. These findings are also consistent with those of Deal and colleagues, who reported lower inter-item correlations and convergent validity at baseline than end-of-study [1]. Studies of emotional function/processing in BED have shown high levels of alexithymia (inability to identify and describe emotions), impaired interoceptive awareness (ability to recognize and respond to emotional states and visceral sensations), and impaired emotion regulation, including low cognitive reappraisal $[18,19]$. These findings suggest that an inability to identify and describe emotions and visceral states may negatively impact self-awareness of BED and its severity in individuals with BED. Therefore, it is possible that the weaker baseline psychometric properties of the Y-BOCS-BE are related to poor disease insight, including an inability to discriminate BED symptom severity. However, as the studies progressed, participants gained insight into their disorder through LDX treatment effects and the experience of evaluating their symptoms.

Consistent with a previous Y-BOCS-BE validation [1], MCII estimates varied within estimator across scores and within scores across estimators so meaningful agreement on a representative meaningful change estimate could not be established in either short-term efficacy study. The MCII estimates associated with the most reasonable achievement rates (50\% and 63.5\%) were obtained from the $\Delta \mu$-Baseline SD and $\Delta \mu$-Baseline SEM estimators, respectively. Therefore, the estimates in score reductions of 12 to 17 points were taken to represent the best estimates of clinically meaningful improvement. This estimate range is narrower than the 4- to 
Table 4 Efficacy of LDX on the Y-BOCS-BE scores, FAS

\begin{tabular}{llllr}
\hline Contrast (LDX vs placebo) & $\begin{array}{l}\text { LS mean } \\
\text { contrast }\end{array}$ & $\begin{array}{l}\text { LS mean } \\
95 \% \text { CI }\end{array}$ & $P$ value & $\begin{array}{l}\text { Semipar- } \\
\text { tial } \omega^{2}(\%)\end{array}$ \\
\hline Study 1 & & & $<0.0001$ & 29.8 \\
Overall & & & $<.1$ \\
Change from baseline to Wk 4 & -6.53 & $-8.09,-4.98$ & $<0.0001$ & 11.2 \\
Change from baseline to Wk 8 & -6.94 & $-8.43,-5.44$ & $<0.0001$ & 12.2 \\
Change from baseline to Wk 12 & -7.40 & $-8.93,-5.88$ & $<0.0001$ & \\
Study 2 & & & & 98.4 \\
Overall & & & $<0.0001$ & 0.2 \\
Change from baseline to Wk 4 & -7.11 & $-8.65,-5.56$ & $<0.0001$ & 0.2 \\
Change from baseline to Wk 8 & -7.56 & $-9.16,-5.96$ & $<0.0001$ & 0.3 \\
Change from baseline to Wk 12 & -7.95 & $-9.53,-6.36$ & $<0.0001$ & \\
Study 3 & & & & 29.3 \\
Overall & & & $<0.0001$ & 12.4 \\
Change from RWB to Wk 16 & -5.07 & $-6.57,-3.57$ & $<0.0001$ & 14.9 \\
Change from RWB to Wk 20 & -6.40 & $-8.14,-4.65$ & $<0.0001$ & 14.2 \\
Change from RWB to Wk 24 & -5.16 & $-6.61,-3.72$ & $<0.0001$ & 18.5 \\
Change from RWB to Wk 28 & -6.15 & $-7.66,-4.64$ & $<0.0001$ & 10.7 \\
Change from RWB to Wk 32 & -4.42 & $-5.84,-3.00$ & $<0.0001$ & 12.3 \\
Change from RWB to Wk 38 & -5.58 & $-7.25,-3.90$ & $<0.0001$ & \\
\hline
\end{tabular}

$F A S$ full analysis set, $L D X$ lisdexamfetamine dimesylate, $L S$ least squares, $R W B$ randomized-withdrawal baseline (Wk 12), Wk week, Y-BOCS-BE Yale-Brown Obsessive Compulsive Scale modified for Binge Eating 17-point range reported by Deal and colleagues [1]. While a meaningful change estimate of up to 17 points is large relative in regard to the potential change score range $( \pm 40)$, in fact, this reflects only $21 \%$ of the total range. Moreover, given the large treatment effect of LDX, lesser meaningful change estimates produced achievement rates of $\geq 80 \%$.

Examination of CDF curves for all scores displayed substantial separation between LDX and placebo, with estimated treatment effects being significant in all three studies and consistent with previous publications [6,8]. The observed treatment effects accounted for a substantial percentage of the change score variance for all scores. Based on these findings, $\Delta \mu$-Baseline SEM yielded a narrower CI range, and would be the recommended metric for defining responders in a clinical trial.

Several limitations should be considered when interpreting these data. First, including only participants with protocol-defined moderate-to-severe BED without psychiatric comorbidities limits the generalizability of these findings to the more heterogeneous general population of individuals with BED. Second, although the Eating Disorder Examination Questionnaire is considered a gold standard for assessing eating disorder symptoms, it was not used to validate the Y-BOCS-BE because it was not assessed at the baseline or post-baseline visits. Third, MCII estimators based on standardized data displayed increased homogeneity and may be advantageous in educational testing and real-world evidence contexts, for which larger sample sizes can be used to generate standardized estimates for the purposes of comparison. However, they are sample dependent and do not permit individual patient benchmarking and thus may be impractical in clinical practice due to the need to focus on patient-centric and individualized assessment in the clinical practice setting. Last, when considering treatment effects in the maintenance-of-efficacy study, it is a limitation that the analysis only included participants who did not relapse during the randomized-withdrawal phase.

These analyses from large placebo-controlled studies of LDX indicate that the Y-BOCS-BE is a valuable tool for assessing BED symptoms. These analyses demonstrated that the Y-BOCS-BE can be decomposed into three distinct domains (obsessive/compulsive, restraint, and control) rather than the previously hypothesized two domains of the Y-BOCS-BE. However, a bifactor solution of the threedomain structure best fits the data and our findings were consistent with the local dependence findings contained in the supplemental material of Deal et al. [1]. Since the psychometric properties of the Y-BOCS-BE were substantially stronger at later study time points than at baseline, it may be valuable for clinicians to implement regular self-reports of $\mathrm{BE}$ thoughts and behaviors into their clinical practices in order to enhance patient insight into BED and possibly improve long-term outcomes. Our findings based on MCII estimators for Y-BOCS-BE total score and standardized 
scores set the stage for normalizing the Y-BOCS-BE and increasing the understanding of the clinical significance of Y-BOCS-BE scores and score changes to be useful both for clinical practice and clinical research.

Acknowledgements Under the direction of the authors, Craig Slawecki, PhD, an employee of Complete Healthcare Communications, LLC (CHC; North Wales, PA), a CHC Group company, provided writing and formatting assistance for this manuscript. Shire Development LLC, Lexington, MA, a member of the Takeda group of companies, provided funding to $\mathrm{CHC}$ for support in writing and editing this manuscript.

Funding These clinical studies were funded by Shire Development LLC, Lexington, MA, a member of the Takeda group of companies. These analyses were partially conducted by Dr. Serrano while employed at Endpoint Outcomes (Boston, MA), with funding from Shire Development LLC, a member of the Takeda group of companies. Analyses required to respond to reviewer comments were completed while Dr. Serrano was employed at Pharmerit International (Bethesda, MD).

\section{Compliance with ethical standards}

Conflicts of interest Dr. Yee is an employee of Shire, a member of the Takeda group of companies, and holds Takeda stock. Dr. Kando is a former employee of Shire, a member of the Takeda group of companies, and holds Takeda stock and Johnson \& Johnson stock and/or stock options; she is currently an employee of Tris Pharma (Monmouth Junction, NJ). Dr. Serrano is a former employee of Endpoint Outcomes (Boston, MA); he is currently an employee of Pharmerit International (Bethesda, MD). Dr. McElroy is a consultant to or member of the scientific advisory boards of Avanir, Bracket, F. Hoffmann-La Roche Ltd., Ironshore, Mitsubishi Tanabe Pharma America, Myriad, Novo Nordisk, Otsuka, Shire, and Sunovion. She is a principal or co-investigator on studies sponsored by Allergan, Avanir, Azevan, Brainsway, Marriott Foundation, Medibio, Myriad, National Institute of Mental Health, Novo Nordisk, Shire, and Sunovion. She has been a principal investigator on a study supported by Orexigen Therapeutics. She is also an inventor on US Patent No. 6323,236 B2 (Use of sulfamate derivatives for treating impulse control disorders), and along with the patent's assignee, University of Cincinnati, Cincinnati, $\mathrm{OH}$, has received payments from Johnson \& Johnson, which has exclusive rights under the patent.

Ethical approval The analyses conducted for this study are based on clinical data from three phase 3 studies. For all studies, protocols were approved by ethics committees and conducted in accordance with International Conference on Harmonisation Good Clinical Practice and the principles of the Declaration of Helsinki.

Informed consent All study participants were required to provide written, informed consent before entering the studies.

Open Access This article is distributed under the terms of the Creative Commons Attribution 4.0 International License (http://creativeco mmons.org/licenses/by/4.0/), which permits unrestricted use, distribution, and reproduction in any medium, provided you give appropriate credit to the original author(s) and the source, provide a link to the Creative Commons license, and indicate if changes were made.

\section{References}

1. Deal, L. S., Wirth, R. J., Gasior, M., Herman, B. K., \& McElroy, S. L. (2015). Validation of the Yale-Brown Obsessive Compulsive Scale modified for Binge Eating. International Journal of Eating Disorders, 48(7), 994-1004.

2. Goodman, W. K., Price, L. H., Rasmussen, S. A., Mazure, C., Fleischmann, R. L., Hill, C. L., et al. (1989). The Yale-Brown Obsessive Compulsive Scale. I. Development, use, and reliability. Archives of General Psychiatry, 46(11), 1006-1011.

3. McElroy, S. L., Arnold, L. M., Shapira, N. A., Keck, P. E., Jr., Rosenthal, N. R., Karim, M. R., et al. (2003). Topiramate in the treatment of binge eating disorder associated with obesity: A randomized, placebo-controlled trial. American Journal of Psychiatry, 160(2), 255-261.

4. McElroy, S. L., Guerdjikova, A., Kotwal, R., Welge, J. A., Nelson, E. B., Lake, K. A., et al. (2007). Atomoxetine in the treatment of binge-eating disorder: A randomized placebo-controlled trial. Journal of Clinical Psychiatry, 68(3), 390-398.

5. McElroy, S. L., Hudson, J. I., Capece, J. A., Beyers, K., Fisher, A. C., \& Rosenthal, N. R. (2007). Topiramate for the treatment of binge eating disorder associated with obesity: A placebocontrolled study. Biological Psychiatry, 61(9), 1039-1048.

6. McElroy, S. L., Hudson, J., Ferreira-Cornwell, M. C., Radewonuk, J., Whitaker, T., \& Gasior, M. (2016). Lisdexamfetamine dimesylate for adults with moderate to severe binge eating disorder: Results of two pivotal phase 3 randomized controlled trials. Neuropsychopharmacology, 41(5), 1251-1260.

7. McElroy, S. L., Hudson, J. I., Mitchell, J. E., Wilfley, D., Ferreira-Cornwell, M. C., Gao, J., et al. (2015). Efficacy and safety of lisdexamfetamine for treatment of adults with moderate to severe binge-eating disorder: A randomized clinical trial. JAMA Psychiatry, 72(3), 235-246.

8. Hudson, J. I., McElroy, S. L., Ferreira-Cornwell, M. C., Radewonuk, J., \& Gasior, M. (2017). Efficacy of lisdexamfetamine in adults with moderate to severe binge-eating disorder: A randomized clinical trial. JAMA Psychiatry, 74(9), 903-910.

9. Gormally, J., Black, S., Daston, S., \& Rardin, D. (1982). The assessment of binge eating severity among obese persons. Addictive Behaviors, 7(1), 47-55.

10. Stunkard, A. J., \& Messick, S. (1985). The Three-Factor Eating Questionnaire to measure dietary restraint, disinhibition and hunger. Journal of Psychosomatic Research, 29(1), 71-83.

11. Guy, W. (1976). ECDEU assessment manual for psychopharmacology. Rockville, MD: National Institute of Mental Health, Psychopharmacology Research Branch.

12. Schafer, J. L. (1999). Multiple imputation: A primer. Statistical Methods in Medical Research, 8(1), 3-15.

13. Rodriguez, A., Reise, S. P., \& Haviland, M. G. (2016). Evaluating bifactor models: Calculating and interpreting statistical indices. Psychological Methods, 21(2), 137-150.

14. Browne, M. W. (2001). An overview of analytic rotation in exploratory factor analysis. Multivariate Behav Res, 36(1), 111-150.

15. Nesselroade, J. R., \& Baltes, P. B. (1984). From traditional factor analysis to structural causal modeling in developmental research. In V. S. A. Parducci (Ed.), Perspectives in psychological experimentation: Toward the year 2000. Hillsdale, NJ: Erlbaum.

16. Gibbons, R. D., \& Hedecker, D. R. (1992). Full-Information item bi-factor analysis. Psychometrika, 57(3), 423-436.

17. Harvill, L. M. (1991). Standard error of measurement. Educational Measurement: Issues and Practice, 10(2), 33-41.

18. Aloi, M., Rania, M., Caroleo, M., De Fazio, P., \& Segura-Garcia, C. (2017). Social cognition and emotional functioning in patients 
with binge eating disorder. European Eating Disorders Review, 25(3), 172-178.

19. Fernandes, J., Ferreira-Santos, F., Miller, K., \& Torres, S. (2018). Emotional processing in obesity: A systematic review and exploratory meta-analysis. Obesity Reviews, 19(1), 111-120.
Publisher's Note Springer Nature remains neutral with regard to jurisdictional claims in published maps and institutional affiliations. 